\title{
Two-Generation Confirmation of Crown-Size Body-Size Relationships in Human Beings
}

\author{
STANLEY M. GARN, ARTHUR B. LEWIS, and ARLINE J. WALENGA \\ Center for Human Growth, University of Michigan, Ann Arbor, Michigan and Fels Re- \\ search Institute, Yellow Springs, Ohio
}

\begin{abstract}
Although the existence of a relationship between crown size and body size has previously been questioned (S. M. GARN, and A. B. LEwIS, Aner Anthrop 60:894-880, 1958; H. A. BAILIT and J. S. Friedlaender, Amer Anthrop 68:665$672,1966)$, recent studies show that there are systematic, positive, but low-order, correlations between crown size and various body dimensions (R. FILIPSSON and L. Goldson, Acta Odont Scand 21:359-365, 1963; S. M. GARN ET AL, Arch Oral Biol 13:129, 1968). The question then arises as to whether such relationships are purely developmental, operating only within individuals, or whether they persist over the generations and have a genetic basis.

Correlating the standard height of 137 parents (66 fathers and 71 mothers) with crown sizes of 117 of their offspring ( 60 sons and 57 daughters), it is clear that taller parents do have children with systematically larger mesiodistal and buccolingual dental crown dimensions. This was true for all classes of teeth
\end{abstract}

This research was supported by USPHS Grants DE01294 and FR-00222 from the National Institutes of Health, Bethesda, Md.

Additional infermation is available on request to the authors.

Received for publication June $5,1958$. and for both jaws. In all, 44 out of 112 sex-, tooth-, and dimension-specific correlations were significant at $p=0.05$ or better, in contrast to the five correlations that would be expected by chance. Father-son and father-daughter correlations for body size versus crown size were systematically higher than were corresponding mother-son and mother-daughter correlations, however, and included a larger number that are significant at $p=0.05$ or better $(19$ and 14 versus seven and four, $\chi_{2}=12.5$ against the chance 11:11:11:11 hypothesis).

In this study, aging effects on stature were eliminated by the use of a narrow parental age range. There was minimum spread of socioeconomic classes, and the population sample exceeded most USA stature norms (S. M. GARN and C. G. Rohmann, Pediat Clin N Amer 13:359-379, 1966). The two-generation findings therefore indicated that stature in one generation and dental crown size in the next are slightly, but significantly, related within and across sexes. Idiosyncratic, prenatal, placental, and nutritional effects therefore may be excluded. The hierarchy of communality values, indicating greater dimensional relationships between fathers and their progeny than between mothers and their sons or daughters, cannot be explained by any known mode of inheritance, however.

Parkntal Hody Size anis Chonne Diamethrs of Thetr Cimlloren

\begin{tabular}{|c|c|c|c|c|c|c|c|c|c|}
\hline \multirow[b]{2}{*}{$|\operatorname{los} x|_{2}$} & \multirow[b]{2}{*}{ Diatmetiti } & \multicolumn{4}{|c|}{ 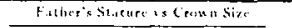 } & \multicolumn{4}{|c|}{ Mutliez's Sexture is Lromen Sixe } \\
\hline & & \multicolumn{2}{|c|}{ Surs, } & \multicolumn{2}{|c|}{ Deashaters } & \multicolumn{2}{|r|}{ Strons } & \multicolumn{2}{|c|}{ Datighiers } \\
\hline $\mathrm{I}^{2}$ & buccolingual & 110 & $0.33^{*}$ & 109 & 0.16 & 116 & 0.16 & 113 & $\overline{0.02}$ \\
\hline & mesiodistal & 113 & 0.13 & 97 & 0.13 & 119 & -0.02 & $9 ?$ & 0.01 \\
\hline \multirow[t]{2}{*}{$j^{2}$} & buccolingual & 106 & $0.55^{*}$ & $10 \mathrm{H}$ & -0.03 & i11 & $0.34^{*}$ & 105 & 0.00 \\
\hline & mesiodistal & 111 & $0.27 *$ & 95 & -0.07 & 117 & 0.19 & 95 & -0.16 \\
\hline \multirow[t]{2}{*}{ C } & buccolingual & 75 & 0.50 : & 76 & 0.04 & 77 & 10.03 & 79 & -0.07 \\
\hline & mesiodistal & 89 & $0.15^{\circ}$ & 79 & $0.32^{*}$ & 94 & 0.12 & 79 & 0.14 \\
\hline \multirow[t]{2}{*}{ P! } & buccolingual & 107 & 1). $40^{*}$ & 100 & $0.38^{*}$ & 113 & $0.23^{*}$ & I04 & $0.26^{\circ}$ \\
\hline & mesiodista! & 164 & 0.11 & 92 & $0.33^{*}$ & 110 & $0.20^{*}$ & 92 & $0.2 .4 *$ \\
\hline \multirow[t]{2}{*}{$p$} & buccolingual & 107 & $0.49^{*}$ & 89 & 0.04 & 113 & 0.16 & 93 & 0.12 \\
\hline & mesiodistal & 99 & 0.10 & 77 & $\{0,3\}^{*}$ & If15 & $0.20=$ & 77 & $0.23 *$ \\
\hline \multirow[t]{2}{*}{$M^{1}$} & buccolingua! & $\$ 13$ & $0.24 *$ & 1166 & $0.33^{*}$ & 119 & -0.04 & 110 & 0.08 \\
\hline & mesiodistal & 109 & 0.09 & 94 & $0.29^{*}$ & 15 & 0.14 & 94 & 0.06 \\
\hline \multirow[t]{2}{*}{$M^{2}$} & buccolingual & 67 & $0,47^{*}$ & 55 & $0.40^{\circ}$ & 69 & $0.28^{*}$ & 56 & 0.15 \\
\hline & mesiodistal & 68 & 0.09 & 56 & 001 & 64 & 0.15 & 56 & 0.03 \\
\hline$I_{4}$ & buccolingual & 104 & $0.43^{*}$ & 106 & 0.01 & 119 & $0.25^{*}$ & 110 & -0.05 \\
\hline & mesiodista: & $i 0 y$ & $0.22=$ & 97 & $0.23^{*}$ & 115 & 0.08 & 9) & 0.07 \\
\hline \multirow[t]{2}{*}{$\mathrm{I}_{2}$} & buccolingual & 107 & $0.27^{*}$ & $10 x$ & 0.16 & 113 & $(0,0)$ & 112 & -0.05 \\
\hline & mesiodistal & 111 & $0.25 *$ & 94 & $0.25^{\circ}$ & 117 & 0,03 & 94 & $0.20^{\circ}$ \\
\hline \multirow[t]{2}{*}{ C } & buccolingual & 97 & $0.39 *$ & 94 & -0.04 & 103 & 10.02 & 97 & -0.13 \\
\hline & $\begin{array}{l}\text { mesiodisla! } \\
\text { mesion }\end{array}$ & 105 & $0.30^{*}$ & 91 & $0.24^{*}$ & 111 & -10.08 & 91 & $\begin{array}{r}-0.13 \\
0.08\end{array}$ \\
\hline \multirow[t]{2}{*}{$P_{1}$} & buccolingual & 108 & $0.50^{*}$ & 101 & $0.24^{*}$ & 114 & 1107 & 105 & 0.16 \\
\hline & mesiodistal & 103 & $0.45^{*}$ & 92 & $0.25^{*}$ & $I f x$ & $0.25 *$ & 92 & 0.11 \\
\hline \multirow[t]{2}{*}{$P_{2}$} & buccolingual & 99 & $0.58+$ & $\times 3$ & $0.26^{\circ}$ & 105 & 0.05 & 86 & 0.08 \\
\hline & mesiodistal & 97 & 0.04 & 83 & 0.14 & 10.3 & -0.11 & 83 & $\begin{array}{l}-0.07 \\
-0.07\end{array}$ \\
\hline \multirow[t]{2}{*}{$M_{1}$} & buccolingual & .07 & $0.34^{*}$ & 107 & $0.27 *$ & 113 & -0.18 & Iii & -0.17 \\
\hline & mesiodistal & 103 & 0.11 & 84 & 0.01 & ing & -0.05 & 84 & 0.09 \\
\hline \multirow[t]{2}{*}{$M_{2}$} & buccoljngual & 47 & $0.57^{\circ}$ & 41 & 0.25 & 49 & 0.25 & 41 & -0.24 \\
\hline & & 54 & 0.08 & 34 & -0.17 & 56 & 0.21 & 39 & -0.18 \\
\hline \multicolumn{2}{|c|}{ No. of significant ris } & & 19 & & 14 & & 7 & & \\
\hline
\end{tabular}

